\title{
A SINGLE REFERENCE CENTER, 2 YEARS RETROSPECTIVE STUDY ON THE RISK FACTORS ASSOCIATED WITH ANTI-RESORPTIVE MEDICATION- RELATED OSTEONECROSIS OF THE JAWS IN OSTEOPOROTIC AND ONCOLOGICAL PATIENTS
}

\author{
Pamela Pignatelli ${ }^{1}$, Gian Marco Podda, daniele pergolini ${ }^{1}$, FEDERICA ROCCHETTI ${ }^{1}$, umberto romeo ${ }^{1}$, Gianluca \\ Tenore $^{1}$, Domenico Gaglioti
}

1 Sapienza University of Rome

Funding: The author(s) received no specific funding for this work.

Potential competing interests: The author(s) declared that no potential competing interests exist.

\begin{abstract}
Medication-related osteonecrosis of the jaws (MRONJ) has multiple exogenous and endogenous risk factors. The risk of MRONJ associated with anti-resorptive drugs (Bisphosphonates and Denosumab) was reviewed in oncological and osteoporotic patients referred to the Department of Oral and Maxillo-Facial Sciences, Sapienza University of Rome (Italy), since January 2019 to January 2021. The incidence of MRONJ was analyzed according to local (dental extraction), systemic risk factors (diabetes, steroid, ecc), medication type, and drug treatment duration. The intake of Zolendronate was strongly associated with osteonecrosis of the jaws in oncological patients and the risk of MRONJ was higher in patients with metastases regardless of the type of anti-resorptive therapy. The use of Bisphosphonates and Denosumab was very common in the treatment of osteoporosis, up to $42.4 \%$ of the patients, and the incidence of MRONJ was higher than previously reported. It was possible to prevent the occurrence of MRONJ after oral surgery due to medical prophylaxis, non-traumatic surgical procedures, and photobiomodulation therapy (PBMT).
\end{abstract}

Introduction

Medication-related osteonecrosis of the jaws (MRONJ) is a potentially severe adverse effect of anti-resorptive and antiangiogenic therapies. There are multiple exogenous and endogenous risk factors that favor MRONJ. Among the exogenous factors are included the type of antiresorptive drug, smoking habit, concomitant pharmacological therapies (i.e., glucocorticoid, calcineurin-inhibitors, particularly cyclosporine). Dentoalveolar trauma (i.e., tooth extraction or dental surgery, infection, or implant), denture use, poor oral hygiene, cumulative dose and length of exposure to anti-resorptive seem to be the most important risk factors for MRONJ ${ }^{[1]}$. Endogenous risk factors have also been identified including systemic diseases such as diabetes or hypertension and adverse polymorphisms of genes involved in metabolism such as CYPs and MTHFR ${ }^{[2]}$. The aim of this study was to analyze the clinical experience of a reference medical center for oral 
osteonecrosis and to assess the risk factors associated with anti-resorptive therapy (Bisphosphonates and Denosumab).

Materials and Methods

A retrospective analysis was carried out on patients referred to the Department of Oral and Maxillo-Facial Sciences, Sapienza University of Rome (Italy), since January 2019 to January 2021; patients treated with anti-resorptive drugs (bisphosphonates and denosumab) were selected for the present study. Only a little number of patients were treated with antiangiogenic medications; therefore, they were not considered in the analysis. Patients with insufficient data on medication-related risk factors were excluded.

In all cases, MRONJ diagnosis and staging were made combining medical history, clinical and radiological ( Panoramic radiograph and Computed Tomography ) examination following the SIPMO/SICMF staging classification [3] [4]. Demographic and clinical variables (sex, age, primary disease, medication type, mode of delivery, anatomic location, drug treatment duration, tooth extraction, chemotherapy, disease stage, and treatment modality) were examined in all the patients. The occurrence rate of MRONJ was calculated both for osteoporotic and oncological patients. The relationship between clinical factor (diabetes, steroid, ecc), medication type, and osteonecrosis jaw was analyzed using $\chi^{2}$ test of association and Odds ratio (OR) calculation; if necessary, the Haldane-Anscombe correction was applied. The positive predictive value, as the probability of developing MRONJ given a condition (e.g., use of drugs), and the negative predictive value, as the probability of not developing MRONJ in absence of the previous condition, were also calculated. In case of a positive association, Event History analysis was applied considering the time elapsed from each treatment onset and MRONJ occurrence. Analyses were carried out using the R-based open-source software Jamovi Version 1.6.18.0 (retrieved from https://www.jamovi.org).

Results

A total sample of 170 patients (149 females and 21 males, aged $69.28 \pm 11.96$ years) with history of anti-resorptive therapies were included. The use of anti-resorptive drugs was very common in the treatment of osteoporosis, even $42.4 \%$ $(n=72)$ of the patients referring to our Department. The incidence of MRONJ in patients treated for osteoporosis was $16.9 \%(n=12)(95 \% \mathrm{Cl}: 9.0 \%-27.7 \%)$. The $57.6 \%$ of cohort was constituted by oncological patients, of whom $41.8 \%(n=$ 41) (95\% Cl: $31.9 \%$ - 52.2\%) patients (22 for breast cancer, 5 for prostate cancer, 6 for lung cancer, 2 for kidney cancer, 2 for thyroid cancer, 2 for multiple myeloma, and 2 for melanoma) developed MRONJ. Total bone sites affected were 66 (maxilla:22 ; mandible:44). In 3 cases MRONJ was associated with dental implants. According to the staging system SICMF-SIPMO, 27 cases were classified as stage I, 20 as stage 2, and 4 cases as stage 3 . The most common drug was Alendronate for the treatment of osteoporosis and Denosumab for cancer therapy.

With regard to the osteoporotic group, the use of Risendronate and Zolendronate was not associated with MRONJ occurrence, likely due to the small number of osteoporotic patients. The percentage of MRONJ occurrence was higher in patients treated with Ibandronate (probability of ONJ occurence was $28.6 \%$ in Ibandronate users vs. $15.6 \%$ in other drugs users), although far from statistical significance $(p=.386, O R=2.16)$. The use of Denosumab had also a positive - 
although not significant - association with MRONJ, (probability of ONJ occurence was $28.6 \%$ in denosumab users vs. $14.0 \%$ in other drugs users, $p=.193, \mathrm{OR}=2.45$ ). The event history analysis revealed the median of time elapsed from treatment onset and MRONJ occurrence was lower in patients treated with Denosumab (30 months) compared to the others (96 months). Instead, the use of Alendronate was not associated with MRONJ occurrence. Treatments taken only by a little number of patients, as steroids, Neridronate, Bevacizumab, Clodronate were not considered in the analysis. In our sample there were few diabetic patients, but they revealed a tendency to a higher risk of osteonecrosis jaw (probability of ONJ occurence was $33.3 \%$ in diabetic patients vs. $15.6 \%$ non diabetic patients, $p=.271$, OR=2.70).

With regard to the oncological group, treatment with Zolendronate was positively associated with MRONJ occurrence ( probability of ONJ occurence was $76.0 \%$ in Zolendronate users vs. $30.1 \%$ in other drugs users, $p<.001, \mathrm{OR}=7.34$ ); in the same vein, a positive association was found between the presence of metastasis and MRONJ occurrence (probability of ONJ occurence was $59.5 \%$ in patients with metastases vs. $28.6 \%$ patients without metastases, $p=.002, O R=3.68$ ); as metastasis was strongly associated with Zolendronate $(p<.001, O R=9.27)$, we controlled the association between metastasis and MRONJ occurrence in patients who did not take Zolendronate, and such association remained positive, although at a lower extent $(p=.061, O R=2.71)$. No association were found between MRONJ occurrence and treatments with Alendronate, Risendronate or Clodronate. Treatments taken only by a little number of patients (or no patients), as Bevacizumab, Neridronate, Ibandronate were not considered in the analysis. Treatment with Denosumab did not show any association with MRONJ occurrence (42.2\% of patients taking the drug developed MRONJ), as for steroid (46.2\% of patients taking the drug developed MRONJ), and the presence of diabetes (30.0\% of patients developed MRONJ). MRONJ development was triggered by tooth extraction, performed in private dental clinics, in 14 (7 osteoporotic and 7 oncological patients) out of 53 cases of osteonecrosis jaw. Instead, 27 patients underwent surgery therapy (tooth extraction) at our Department did not develop MRONJ, thanks to medical prophylaxis $(0.12 \%$ chlorhexidine antiseptic mouthwash used at home 3 times a day, starting from 10 days prior to the dental surgery to 15 days post-surgery, associated with an amoxicillin-clavulanic acid $875 \mathrm{mg}+125 \mathrm{mg}$ and metronidazole $500 \mathrm{mg}$ three times per day, administered from the day before the intervention and for at least 6 days following intervention), strictly non-traumatic surgical procedures, and photobiomodulation therapy (PBMT).

\section{Conclusions}

The use of anti-resorptive drugs for the treatment of osteoporosis is increasingly frequent, in fact our study cohort had $42.4 \%$ of osteoporotic patients. The incidence of MRONJ in our patients with osteoporosis was higher than estimated (0.001-0.01\%) ${ }^{[5]}$. The most osteoporotic patients were treated with Alendronate or Denosumab. Denosumab has been shown to be more effective than zoledronic acid in increasing bone mineral density (BMD), inhibiting bone turnover in postmenopausal women with osteoporosis, and in preventing skeletal-related events in cancer patients with bones metastases [6] [7].

When comparing Denosumab vs Alendronate, Denosumab resulted in better BMD results and a significantly greater reduction in bone turnover markers $(\mathrm{BTMs})^{[8]}$. For this reason its prescription has increased over time. Notwithstanding the numerous advantages in its use, Taylor et al suggested that the incidence of Denosumab-related ONJ may increase in the 
future ${ }^{[9]}$. In fact, our patients using Denosumab to treat osteoporosis were more likely to develop ONJ than users of other antiresorptive drugs. The reported incidence of ONJ denosumab-related for the treatment of osteoporosis is very low [10] [11] . The possibility of MRONJ occurrence was not increased among Denosumab users with cancer. Denosumabrelated ONJ has a faster onset (30 months), perhaps due to the pharmacodynamic of this drug than other anti-resorptives therapies. The risk of ONJ for bisphosphonates intake was dose and time correlated, with a ONJ prevalence of $0.21 \%(21$ cases per 10 000) among patients with more than 4 years' treatment ${ }^{[12]}$, while the MRONJ risk for Denosumab intake would appear to be more related to the dose ( Denosumab does not accumulate in bone and exerts a more transient effect on the inhibition of bone resorption) ${ }^{[13]}$. However, our results referred to a limited number of patients. There was a tendency to an increased risk of ONJ among osteoporotic diabetic patients, unlike what has been reported in the literature ${ }^{[14]}[15]$. The association of diabetes with ONJ pathogenesis was related to impairment of lymphocyte function, microvascular ischemia, endothelial cell dysfunction, reduced remodeling of bone, and increased apoptosis of osteoblasts and osteocytes via Nod-like receptor (NLR) family pyrin domain-containing protein 3 (NLRP3) inflammasome activation in macrophages $^{[16]}$.

In our cancer patients the intake of Zolendronate was strongly associated with ONJ with a higher incidence than previously reported (from $0.7 \%$ to $6.7 \%, 70-670$ cases for 10000 patients) ${ }^{[17]}$. When patients with cancer were treated with Denosumab, the risk of MRONJ ranges from $0.7 \%$ to $1.9 \%$ (70-190 cases per 10000 patients $)^{[18]}$. There was no association between Denosumab and ONJ in our cancer patients. The highest rate of ONJ has been reported in patients with breast cancer. The concomitant use of capecitabine, gemcitabine, paclitaxel, and vinorelbine in breast cancer patients might explain the increased rate of $\mathrm{ONJ}$ in this group ${ }^{[19]}$. Unexpectedly, patients with metastases had a higher risk of MRONJ regardless of the assumption of bone resorption inhibitors. This association could be investigated in a larger study sample. We found that despite cancer patients had a higher risk of MRONJ than osteoporotic patients, the latter had a higher MRONJ incidence than expected. Nevertheless, it was possible to effectively prevent the onset of jaw surgery-related osteonecrosis through a prevention medical protocol and PBMT.

\section{References}

1. ' T. Fehm, V. Beck, M. Banys, H.P. Lipp, et al. (2009). Bisphosphonate-induced osteonecrosis of the jaw (ONJ): Incidence and risk factors in patients with breast cancer and gynecological malignancies. Gynecologic Oncology, vol. 112 (3), 605-609. doi:10.1016/j.ygyno.2008.11.029.

2. 'A, Menini M, Pulliero A, Dini G, Cartiglia C, Pera P, Baldi D.. (2013). Biphosphonates-associated osteonecrosis of the jaw: the role of gene-environment interaction. J Prev Med Hyg., vol. 2013 Sep;54(3):138-45 .

3. ^ Bedogni A, Fusco V, Agrillo A, Campisi G.. (2012). Learning from experience. Proposal of a refined definition and staging system for bisphosphonate-related osteonecrosis of the jaw (BRONJ).. Oral Dis., vol. Sep;18(6):621-3 .

4. 'Bedogni A, Fedele S, Bedogni G, Scoletta M, Favia G, Colella G, Agrillo A, Bettini G, Di Fede O, Oteri G, Fusco V, Gabriele M, Ottolenghi L, Valsecchi S, Porter S, Petruzzi M, Arduino P, D'Amato S, Ungari C, Fung Polly PL, Saia G, Campisi G.. (2014). Staging of osteonecrosis of the jaw requires computed tomography for accurate definition of the extent of bony disease. Br J Oral Maxillofac Surg., vol. Sep;52(7):603-8 .

5. ^Aliya A Khan, Archie Morrison, David A Hanley, Dieter Felsenberg, et al. (2014). Diagnosis and Management of 
Osteonecrosis of the Jaw: A Systematic Review and International Consensus. J Bone Miner Res, vol. 30 (1), 3-23. doi:10.1002/jbmr.2405.

6. 'Allan Lipton, Karim Fizazi, Alison T. Stopeck, David H. Henry, et al. (2012). Superiority of denosumab to zoledronic acid for prevention of skeletal-related events: A combined analysis of 3 pivotal, randomised, phase 3 trials. European Journal of Cancer, vol. 48 (16), 3082-3092. doi:10.1016/j.ejca.2012.08.002.

7. ^ P. D. Miller, N. Pannacciulli, J. P. Brown, E. Czerwinski, et al. (2016). Denosumab or Zoledronic Acid in Postmenopausal Women With Osteoporosis Previously Treated With Oral Bisphosphonates. The Journal of Clinical Endocrinology \& Metabolism, vol. 101 (8), 3163-3170. doi:10.1210/jc.2016-1801.

8. `Jacques P Brown, Richard L Prince, Chad Deal, Robert R Recker, et al. (2009). Comparison of the Effect of Denosumab and Alendronate on BMD and Biochemical Markers of Bone Turnover in Postmenopausal Women With Low Bone Mass: A Randomized, Blinded, Phase 3 Trial*. Journal of Bone and Mineral Research, vol. 24 (1), 153-161. doi:10.1359/jbmr.0809010.

9. 'K.H. Taylor, L.S. Middlefell, K.D. Mizen. (2010). Osteonecrosis of the jaws induced by anti-RANK ligand therapy. British Journal of Oral and Maxillofacial Surgery, vol. 48 (3), 221-223. doi:10.1016/j.bjoms.2009.08.030.

10. 'T. Sugimoto, T. Matsumoto, T. Hosoi, T. Miki, et al. (2014). Three-year denosumab treatment in postmenopausal Japanese women and men with osteoporosis: results from a 1-year open-label extension of the Denosumab Fracture Intervention Randomized Placebo Controlled Trial (DIRECT). Osteoporos Int, vol. 26 (2), 765-774. doi:10.1007/s00198014-2964-2.

11. 'Socrates Papapoulos, Roland Chapurlat, Cesar Libanati, Maria Luisa Brandi, et al. (2012). Five years of denosumab exposure in women with postmenopausal osteoporosis: Results from the first two years of the FREEDOM extension. $J$ Bone Miner Res, vol. 27 (3), 694-701. doi:10.1002/jbmr.1479.

12. 'Joan C. Lo, Felice S. O'Ryan, Nancy P. Gordon, Jingrong Yang, et al. (2010). Prevalence of Osteonecrosis of the Jaw in Patients With Oral Bisphosphonate Exposure. Journal of Oral and Maxillofacial Surgery, vol. 68 (2), 243-253. doi:10.1016/j.joms.2009.03.050.

13. ^Paul D. Miller, Michael A. Bolognese, E. Michael Lewiecki, Michael R. McClung, et al. (2008). Effect of denosumab on bone density and turnover in postmenopausal women with low bone mass after long-term continued, discontinued, and restarting of therapy: A randomized blinded phase 2 clinical trial. Bone, vol. 43 (2), 222-229. doi:10.1016/j.bone.2008.04.007.

14. ^Roman Rahimi-Nedjat, Keyvan Sagheb, Andreas Pabst, Lukas Olk, et al. (2016). Diabetes Mellitus and Its Association to the Occurrence of Medication-Related Osteonecrosis of the Jaw. Dentistry Journal, vol. 4 (2), 17. doi:10.3390/dj4020017.

15. 'A. Shudo, H. Kishimoto, K. Takaoka, K. Noguchi. (2018). Long-term oral bisphosphonates delay healing after tooth extraction: a single institutional prospective study. Osteoporos Int, vol. 29 (10), 2315-2321. doi:10.1007/s00198-0184621-7.

16. 'Qunzhou Zhang, Weihua Yu, Sumin Lee, Qilin Xu, et al. (2015). Bisphosphonate Induces Osteonecrosis of the Jaw in Diabetic Mice via NLRP3/Caspase-1-Dependent IL-1ß Mechanism. J Bone Miner Res, vol. 30 (12), 2300-2312. doi:10.1002/jbmr.2577. 
17. ^R.E. Coleman, E.J. Woodward”, J.E. Brown, D. Cameron, et al. (2011). Safety of zoledronic acid and incidence of osteonecrosis of the jaw (ONJ) during adjuvant therapy in a randomised phase III trial (AZURE-BIG 01-04) for women with stage IIIIII breast cancer. Bone, vol. 48 (1), S29. doi:10.1016/j.bone.2010.10.079.

18. `Wei-Xiang Qi, Li-Na Tang, Ai-Na He, Yang Yao, et al. (2013). Risk of osteonecrosis of the jaw in cancer patients receiving denosumab: a meta-analysis of seven randomized controlled trials. Int J Clin Oncol, vol. 19 (2), 403-410. doi:10.1007/s10147-013-0561-6.

19. ' John H. Wessel, Thomas B. Dodson, Athanasios I. Zavras. (2008). Zoledronate, Smoking, and Obesity Are Strong Risk Factors for Osteonecrosis of the Jaw: A Case-Control Study. Journal of Oral and Maxillofacial Surgery, vol. 66 (4), 625-631. doi:10.1016/j.joms.2007.11.032. 